\title{
A Rare Hyperextension Injury in Thoracic Spine Presenting with Delayed Paraplegia
}

\author{
Dong-Eun Shin, Ki-Sik Nam, Hyung-Ku Yoon, Jun-Ku Lee, Yoon-Sik Cha \\ Department of Orthopaedic Surgery, CHA Bundang Medical Center, CHA University, Seongnam, Korea
}

Hyperextension injury in the thoracic spine is uncommon with only a few cases documented in the literature. The mechanism of these injuries is hyperextension combined with axial or shearing force. These types of injuries are associated with a high risk of dural tears and paraplegia. A 91-year-old female presented with acute back pain from a hyperextension injury in thoracic spine with no neurological deficit. Lumbar magnetic resonance imaging showed a intervertebral disc rupture. On day 20 of hospitalization, the herniated intervertebral disc compressed the spinal cord with incomplete paraplegia. Hyperextension injuries involving the three columns are very unstable and we recommend surgical treatment as soon as possible, not only because of the initial trauma, but a ruptured disc herniation can damage the spinal cord.

Keywords: Thoracic vertebrae; Paraplegia; Disc herniation

\section{Introduction}

Hyperextension injury in the thoracic spine is uncommon and can be caused by direct or indirect trauma [14]. Because of its low incidence, Denis [3] did not include hyperextension injury in the major thoracolumbar spinal injuries. Such injuries can cause rupture of the intervertebral disc, especially the annulus fibrosus, the anterior longitudinal ligament (ALL), and avulsion of a small bone fragment from the anterior border of the dislocated vertebra-this dislocation almost always reduces spontaneously and is stable in flexion $[4,5]$.

We report a case of hyperextension injury in the low thoracic spine, combined with traumatic disc herniation. Because a herniated disc progressively compresses the spinal cord, the patient in our case developed delayed incomplete paraplegia, which is rare and different from previous reports.

\section{Case Report}

A 91-year-old female patient visited our hospital. She was in good condition and walked very well for her age. While she was getting on a bus, she lost her footing and fell backwards onto the ground. She was unable to walk because of severe back pain, but the initial physical examination did not show neurologic deficit. She did not complain of sensory decrease and the lower extremity motor grade was more than grade 4 on both lower legs. The initial plain radiographic study showed a fracture at the anterosuperior portion of the thoracic eleventh (T11) vertebral body (Fig. 1). Also, we suspected oblique fractures at the spinous processes of $\mathrm{T} 9$ and $\mathrm{T} 10$ vertebrae, and therefore conducted computed tomography (CT) to confirm fracture characteristics as we diagnosed (Fig. 2). The L2 vertebral body fracture was diagnosed as an old compression fracture. Because the fracture involved the

Received Dec 22, 2011; Revised May 15, 2012; Accepted Jun 10, 2012

Corresponding author: Ki-Sik Nam

Department of Orthopaedic Surgery, CHA Bundang Medical Center, CHA University,

82 Gumi-ro 173beon-gil, Bundang-gu, Seongnam 463-707, Korea

Tel: +82-31-780-5289, Fax: +82-31-708-3578, E-mail: jg197@naver.com 
posterior column, which are spinous process fractures, magnetic resonance imaging (MRI) was required. On MRI, acute benign fracture was shown at the anterosuperior portion of the T11 vertebral body with hemorrhage of the fracture site (Fig. 3). Hemorrhage within the disc was also seen at the T10 to T11 space. The posterior column including spinous process and interspinous ligament had high signal intensity on T2-weight MRI. The ALL was disrupted with the bony fragment attached. Although the interspinous ligament showed a high signal, the signal intensity within the spinal cord was not increased.

Considering the patient's age and the absence of neurologic deficit at the initial phase, conservative management was chosen. Also, bony lesion, vertebral body and spinous process fractures, were more prominent than soft tissue lesions, and we expected only bony union. On day two of hospitalization, we applied thoraco-lumbo-sacral orthosis and started standing physical therapy including tilt table. On seventh day, follow-up plain radiographic showed slightly reduced the fracture site of the T11 vertebra (Fig. 4). Until day 19, she could stand for 20 minutes but couldn't walk by herself. She did not complain of any neurologic change until then.

On day 20 of hospitalization, she complained of lower extremity weakness, and general lower extremity motor grade was decreased to grade 1 or 2 . Sensory functions may have also been slightly decreased. She said the symptoms gradually aggravated over the last three days and

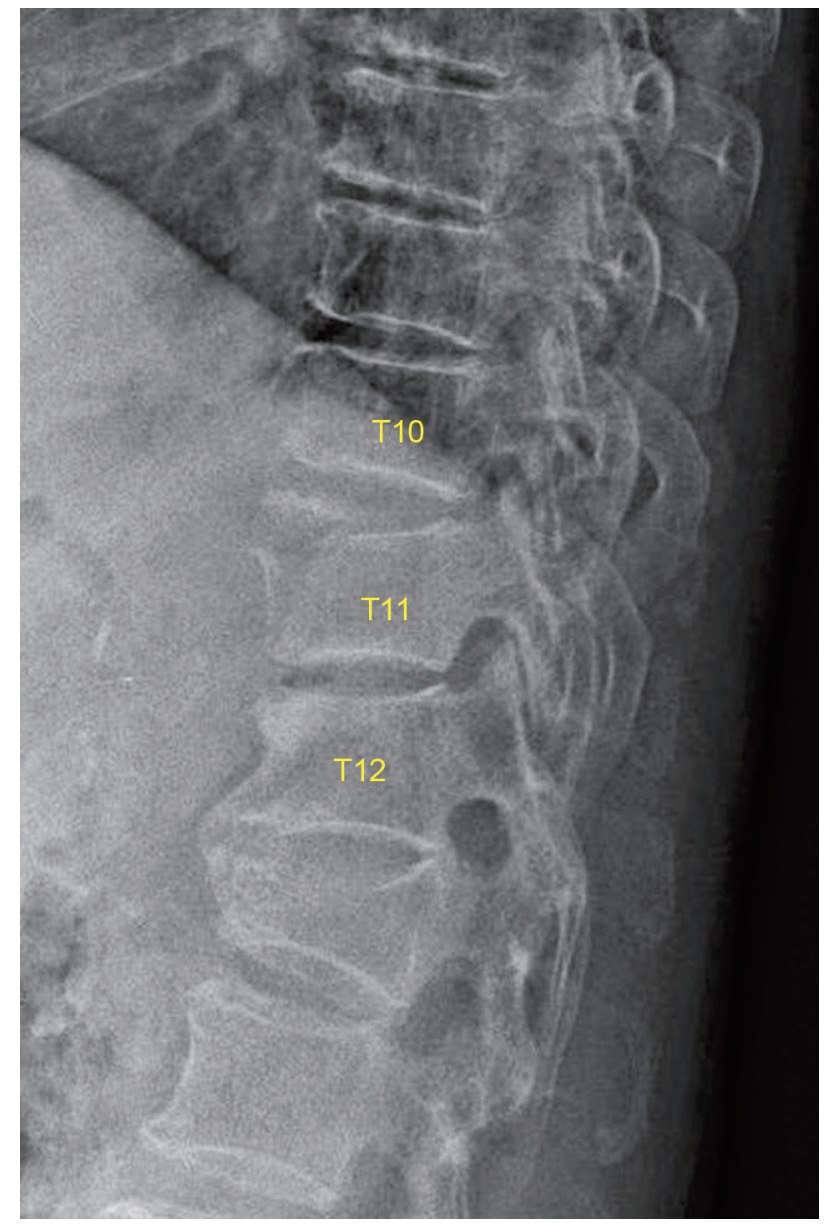

Fig. 1. Lateral radiograph of fracture at the anterosuperior portion of the thoracic eleventh (T11) vertebral body.
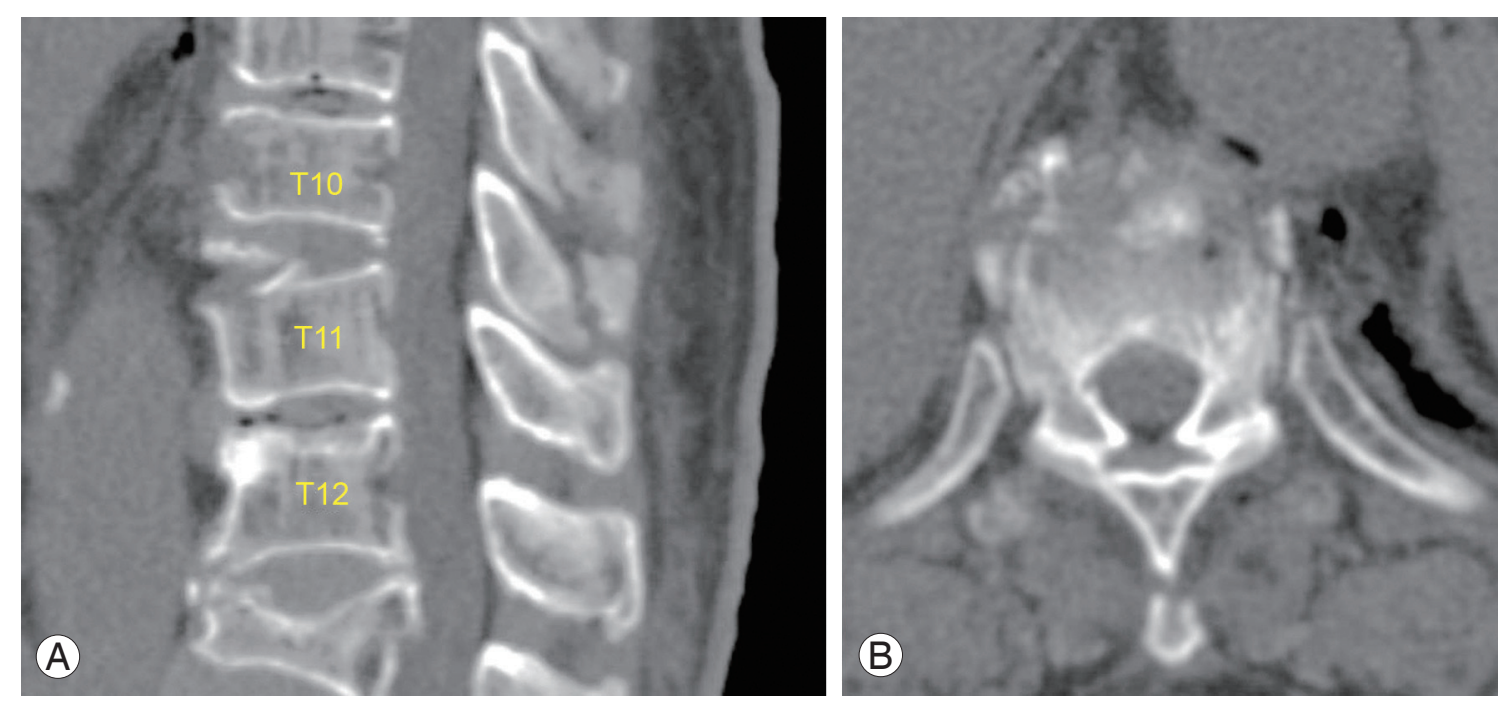

Fig. 2. Initial computed tomography finding. (A) Sagittal image shows fresh fracture at the anterosuperior portion of T11 vertebral body and oblique fractures at the spinous processes of T9 and T10 vertebrae. (B) Axial image shows communicated fracture of T11 anterior column. 
she couldn't remember any significant event before the appearance of the neurologic deficit. Knee jerk and ankle jerk of both lower legs were accelerated. Ankle clonus and babinski sign were positive. We immediately conducted a follow-up plain X-ray and MRI (Fig. 5). The fracture was not more displaced compared to previous imaging, but the intervertebral disc of T10 to T11 space was extruded with superior migration, compressing the spinal cord with compressive myelopathy.

We immediately did surgical decompression (spinous process resection and total laminectomy from T9 to T12), pedicle screws instrumentation and then fusion of T9 to T12 with morcellized allograft (freeze-dried human allograft) and demineralzation bone matrix (Fig. 6). Operation finding showed the dura of the spinal cord was intact. We couldn't remove the herniated disc for fear of further spinal cord injury. Three days after operation we started again standing physical therapy. Three months following surgery, she regained lower extremity motor strength to the grade 4 . She could walk with a walker.

\section{Discussion}

Hyperextension injury was first described in 1970 [6], and several cases have been documented in the literature

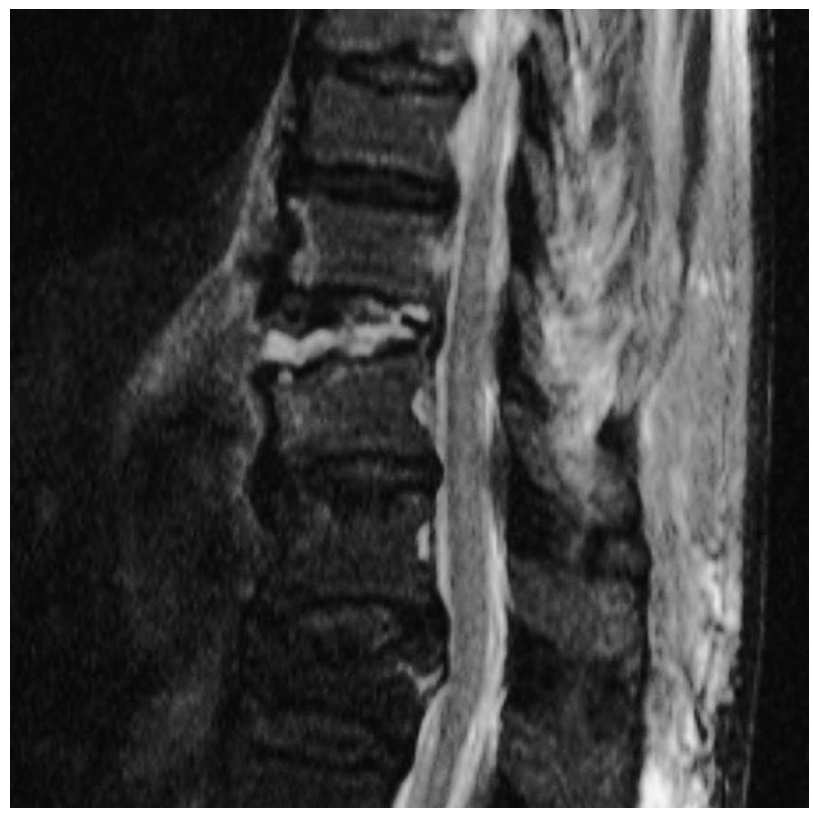

Fig. 3. Initial T2-weighted sagittal magnetic resonance imaging demonstrates acute benign fracture at the anterosuperior portion of T11 vertebral body with hemorrhage of the fracture site and intradiscal hemorrhage at T10 to T11 disc space. The interspinous ligament has high signal intensity.
$[1,2,7]$. Although our case did not show ankylosis of thoracic spine, patients with multiple-level ankylosis of the thoracolumbar spine such as diffuse idiopathic skeletal hyperostosis or ankylosing spondylitis have a higher risk of hyperextension injury in thoracolumbar spine. Loss of compensatory motion of the disc and facet joints adjacent to the area of injury contributes to this fracture pattern [8].

Usually, the mechanism of extension type thoracolumbar fracture was a combination of hyperextension force and axial load [7]. However, De Oliveira [2], Elgafy and Bellabarba [9] report that the combination of hyperextension and posteroanterior shearing force applied on the thoracolumbar spine could create a specific type of injury. In the case of our patient, falling backwards produced a hyperextension force, and landing on her buttocks produced a axial force.

Hyperextension injury is associated with a high risk of dural tears and paraplegia [4].

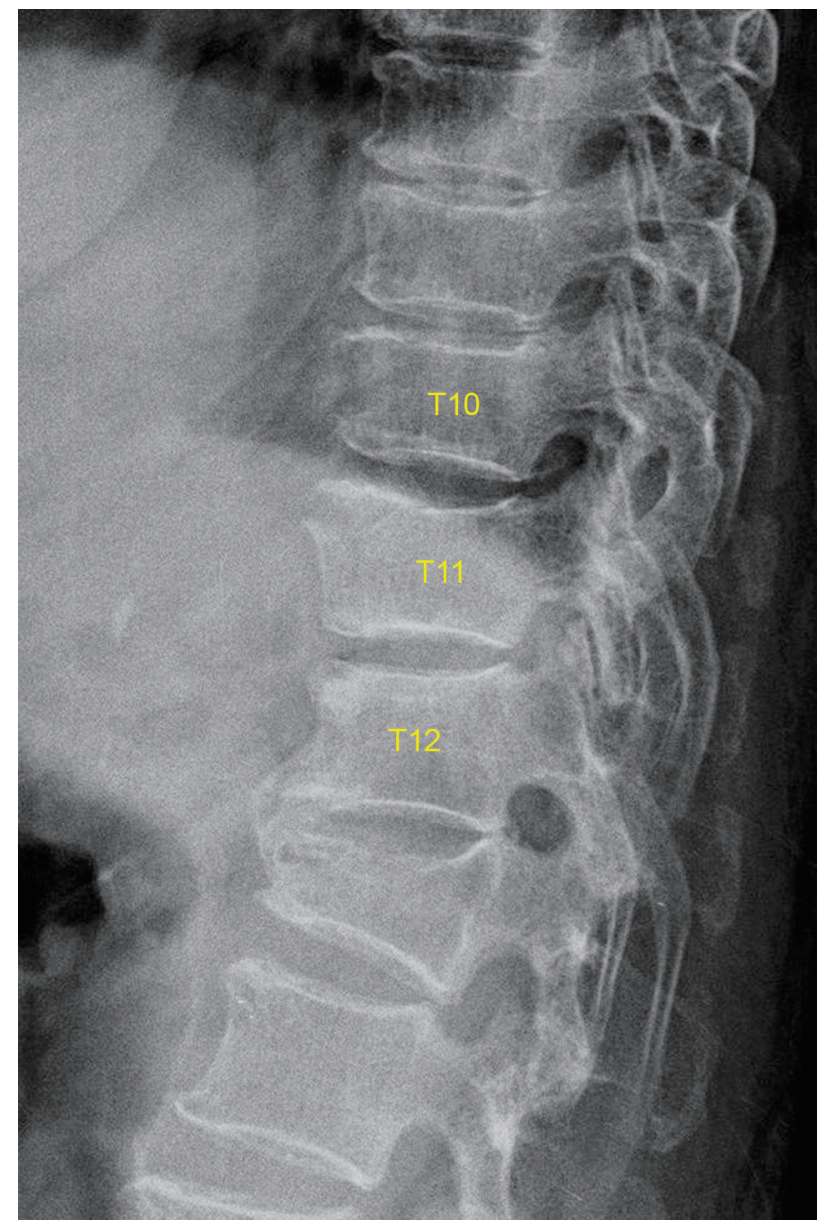

Fig. 4. Follow-up lateral radiograph of the thoracolumbar spine. The fracture site, anterosuperior portion of $\mathrm{T} 11$, is slightly reduced. 
Burke [1] reported that only 4 of 154 cases of thoracolumbar lesions were classified as hyperextension injury. All were in the thoracic spine, and all of the patients with these lesions had complete and permanent paraplegia. Massive spinal cord necrosis had occurred above the level of vertebral injury. This surgical finding indicates that the
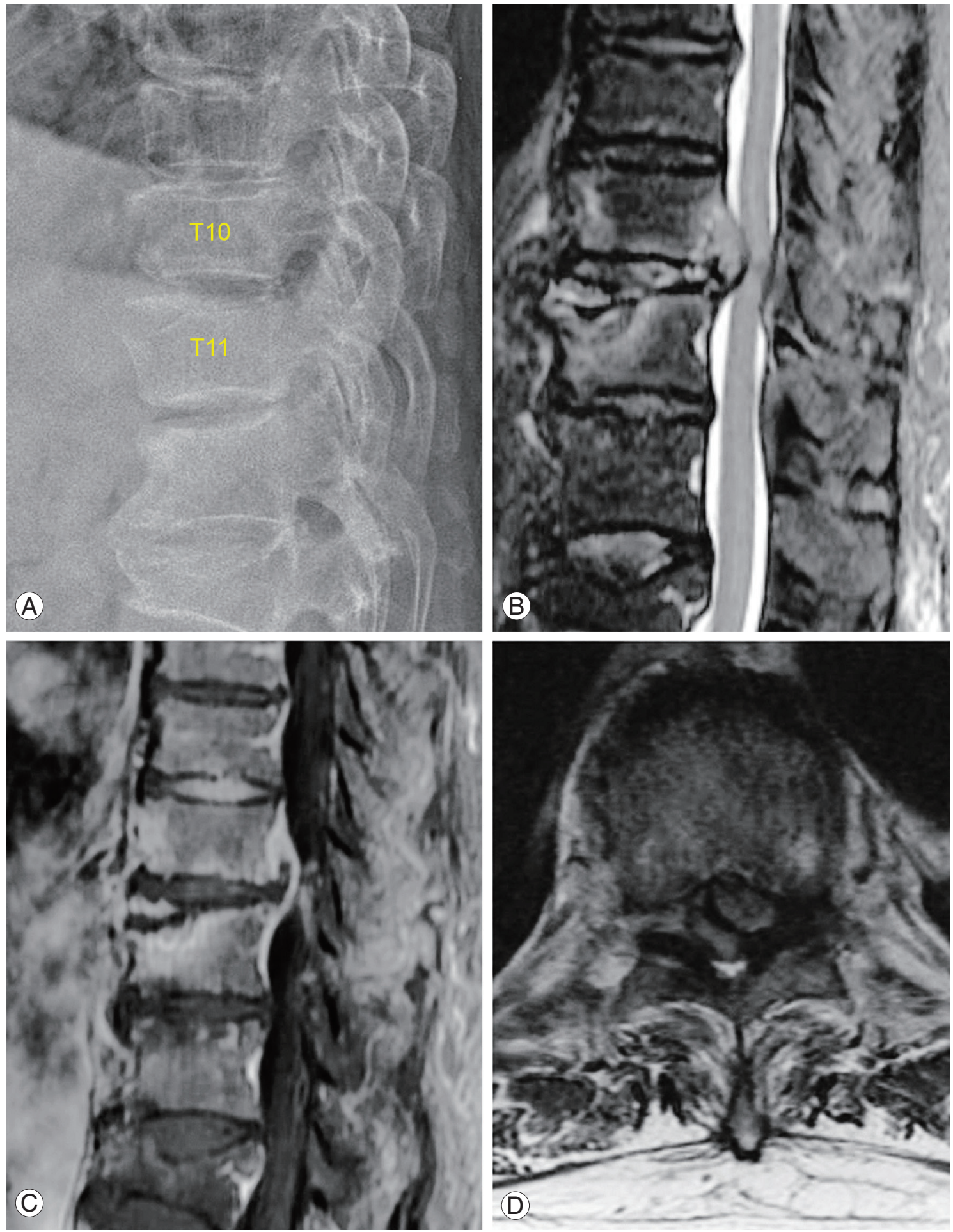

Fig. 5. Follow-up plain lateral X-ray (A) and magnetic resonance imaging. Sagittal T2 weighted imaging (B), sagittal T1 weighted imaging with fat suppression (C) and axial T2 weighted imaging. Central disc extrudes with superior migration at T10 to T11, which compresses the spinal cord with compressive myelopathy. 
mechanism of this spinal cord injury was traction rather than compression.

Denis and Burkus [4] reported 12 patients who sustained a shear fracture-dislocation of their thoracic or lumbar spines by a hyperextension mechanism of injury. During surgery, large dural tears were noted in 6 patients. Three patients had a cord transection, one had a cord hemitransection, and one patient had a lumbar root avulsion.

Nabeshima et al. [7] reported good clinical results with conservative management including closed reduction. $\mathrm{He}$ chose conservative treatment because successful reduction was obtained and maintained by the closed observation and because no neurologic deficit was present.

Disc herniation accompanying spinal fractures and dislocation has been reported infrequently, especially regarding the thoracolumbar spine. Pratt et al. [10] reported herniated intervertebral discs associated with unstable spinal injuries. Twenty-three patients in 93 cases occurred in the thoracolumbar spine. Disc herniations in the thoracolumbar area were less frequent, occurring in 3 of 23 patients as detected by MRI (13\%). They noted a significant risk for disc herniation in unstable spine injuries and recommended MRI as a modality of choice to image the subarachnoid space, disc, and spinal cord in traumatized patients.

The patient was a 91-year-old woman who did not show any evidence of cord injury, such as traction injury at the first presentation. We chose conservative treatment; however, the ruptured disc extruded and compressed the spinal cord. Traumatic disc herniation with paraplegia in thoracic spine is not common; in particular none were reported in extension type injuries as far as we know.

In conclusion, hyperextension injury involving the
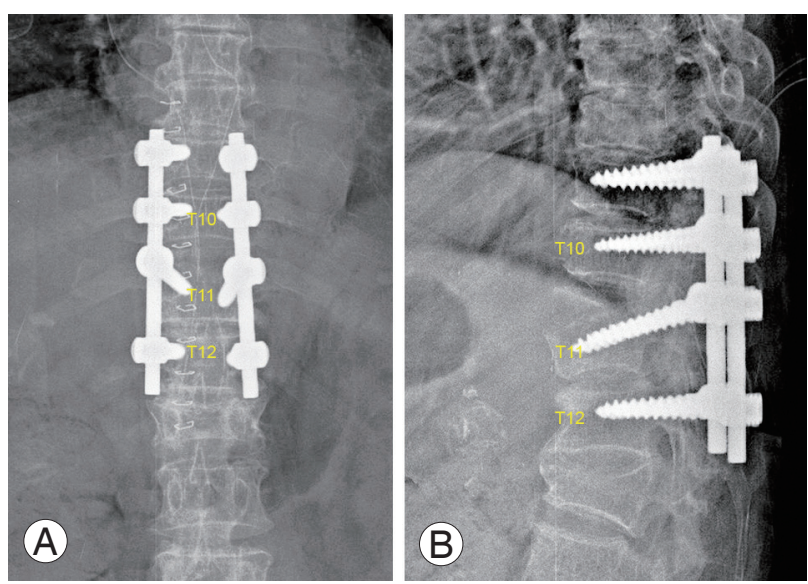

Fig. 6. Postoperative plain anteroposterior (A) and lateral (B) X-ray. three columns is very unstable and we recommend surgical treatment as soon as possible because not only initial trauma such as traction injury but ruptured disc herniation can do damage on the spinal cord.

\section{Conflict of Interest}

No potential conflict of interest relevant to this article was reported.

\section{References}

1. Burke DC. Hyperextension injuries of the spine. J Bone Joint Surg Br 1971;53:3-12.

2. De Oliveira JC. A new type of fracture-dislocation of the thoracolumbar spine. J Bone Joint Surg Am 1978;60:481-8.

3. Denis F. The three column spine and its significance in the classification of acute thoracolumbar spinal injuries. Spine (Phila Pa 1976) 1983;8:817-31.

4. Denis F, Burkus JK. Shear fracture-dislocations of the thoracic and lumbar spine associated with forceful hyperextension (lumberjack paraplegia). Spine (Phila Pa 1976) 1992;17:156-61.

5. Wood GW. Thoracic and lumbosacral fractures. In: Canale ST, Beaty JH, editors. Campbell's operative orthopaedics. Philadelphia, PA: Mosby/Elsevier; 2008. p.1810-30.

6. Holdsworth F. Fractures, dislocations, and fracturedislocations of the spine. J Bone Joint Surg Am 1970;52:1534-51.

7. Nabeshima Y, Iguchi T, Matsubara N, Kinoshita S, Kurosaka M, Mizuno K. Extension injury of the thoracolumbar spine. Spine (Phila Pa 1976) 1997;22:1522-5.

8. Burkus JK, Denis F. Hyperextension injuries of the thoracic spine in diffuse idiopathic skeletal hyperostosis. Report of four cases. J Bone Joint Surg Am 1994;76:237-43.

9. Elgafy H, Bellabarba C. Three-column ligamentous extension injury of the thoracic spine: a case report and review of the literature. Spine (Phila Pa 1976) 2007;32:E785-8.

10. Pratt ES, Green DA, Spengler DM. Herniated intervertebral discs associated with unstable spinal injuries. Spine (Phila Pa 1976) 1990;15:662-6. 